\title{
Humans' Perception of a Robot Moving Using a Slow in and Slow Out Velocity Profile
}

\author{
Trenton Schulz \\ Department of Informatics \\ University of Oslo \\ Oslo, Norway \\ trentonw@ifi.uio.no
}

\author{
Patrick Holthaus \\ School of Computer Science \\ University of Hertfordshire \\ Hatfield, England \\ p.holthaus@herts.ac.uk
}

\author{
Farshid Amirabdollahian \\ School of Computer Science \\ University of Hertfordshire \\ Hatfield, England \\ f.amirabdollahian2@herts.ac.uk
}

\author{
Kheng Lee Koay \\ School of Computer Science \\ University of Hertfordshire \\ Hatfield, England \\ k.1.koay@herts.ac.uk
}

\begin{abstract}
Humans need to understand and trust the robots they are working with. We hypothesize that how a robot moves can impact people's perception and their trust. We present a methodology for a study to explore people's perception of a robot using the animation principle of slow in, slow out-to change the robot's velocity profile versus a robot moving using a linear velocity profile. Study participants will interact with the robot within a home context to complete a task while the robot moves around the house. The participants' perceptions of the robot will be recorded using the Godspeed Questionnaire. A pilot study shows that pilot participants notice the difference between the linear and the slow in, slow out velocity profiles, so the full experiment planned with participants will allow us to compare their perceptions based on the two observable behaviors.

Index Terms-animation; slow in, slow out; velocity profile; home environment;
\end{abstract}

\section{INTRODUCTION}

Robots can help people at home to automate tasks and make it easier for people to live at home independently longer. However, the robots will likely need to interact with humans to accomplish this. These robots need to be trustworthy and something people feel they can understand. Can the way a robot moves during an interaction affect people's perception of the robot and their own feeling of safety?

Robots move using different velocity profiles. Most robots use a velocity profile with linear acceleration (Fig. 1, left). This results in a mechanical, robot-like movement, but a robot could use a different velocity profile. One of the principles of film animation is the idea of slow in, slow out [1]. In slow in, slow out, the movement starts slowly, but then speeds up to its top speed and a slow stop as it reaches its destination (Fig. 1, right). Humans perceive this slow in, slow out acceleration as uniform when compared to linear acceleration [2].

Techniques from animation have been used in other HRI experiments [3]-[5]. We are looking at how a slow in, slow out velocity profile affects a person's perception of the robot. This includes robot's perceived empathy, anthropomorphism, likability, safety, animacy, and intelligence. We are interested if people can notice a difference in how predictable the different types of movement are. We are also interested in collecting people's opinions about how the robot moves.

Partly funded by the Research Council of Norway as part of the Multimodal Elderly Care Systems (MECS) project, under grant agreement 247697.

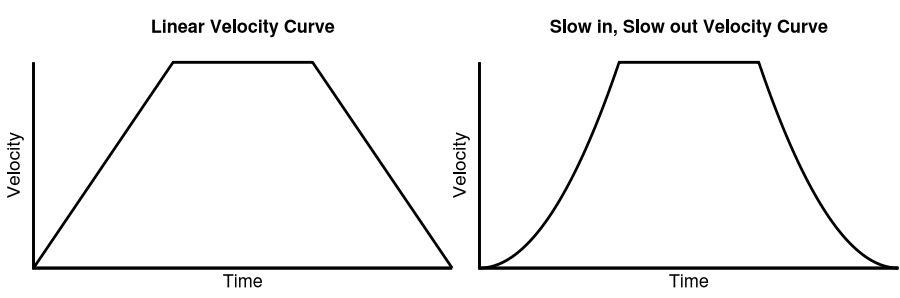

Fig. 1. Most robots use a linear velocity profile (left); the slow in, slow out velocity profile (right) may change people's perception of how robots move.

Our hypothesis are: (HI) Slow in, slow out will positively affect the robot's perceived safety versus linear velocity. $(H 2)$ Slow in, slow out will positively affect the robot's perceived intelligence versus linear velocity. (H3) Slow in, slow out will positively affect the robot's perceived animacy versus linear velocity. (H4) Slow in, slow out will positively affect the robot's perceived anthropomorphism versus linear velocity. (H5) Slow in, slow out will positively affect the robot's perceived likability versus linear velocity.

\section{METHOD}

The hypotheses will be tested by running a within subjects experiment with the variable being the velocity profile the robot uses. We want to see if participants notice the movement when they are interacting with the robot on a task in a home environment. That is, we do not want to influence them to consciously observe the movement, but to see if they notice a difference as they interact with the robot, and if that difference affects their perceptions of the robot. We will be using the Godspeed Questionnaire [6] to measure the participants' perceptions of the robot's intelligence, its animacy, its anthropomorphism, and the participants perceived safety.

For the procedure, a consenting participant will enter the home and have the scenario explained: the participant is visiting a friend's house to help in cleaning up the home. The robot is also helping with the cleaning. We will say we are interested in how the robot handles the hand over of objects. There will be a person sitting on the couch, and a person in the kitchen who are also helping with the clean-up effort.

The participant will stand by a table and give an item to the robot. The robot will then move to the person sitting on the couch. The person on the couch will give a new item 
to the robot. The robot will deliver the items to the kitchen. The person in the kitchen will take the items from the robot and put a copy of the Godspeed Questionnaire that the robot will deliver to the participant to fill out. A map of the hand off and exchange is shown in Fig. 2. This procedure will be repeated three times (four iterations in total). The robot will use a linear velocity curve twice and slow in, slow out curve twice. The ordering of the robot's movement will be counterbalanced to avoid ordering effects. After the final iteration, we ask additional questions concerning the overall interaction. We will also ask participants to watch a video of a person interacting with the same robot using both velocity curves and ask if participants can notice any differences with the robot. This is to see if the participant perceives a difference in the movement when they watch a video of a person working with a robot.

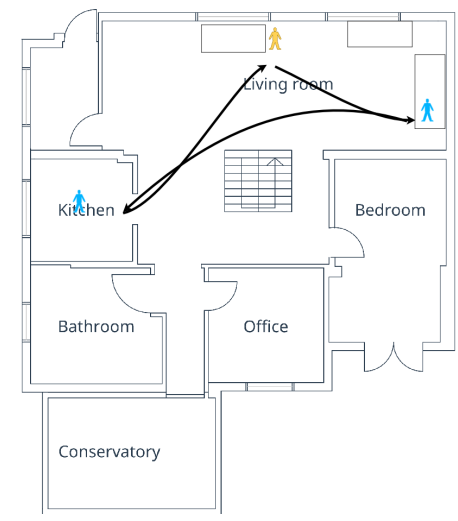

Fig. 2. The experiment set up; the robot will move on the paths in the arrows, the blue people are facilitators, and the orange person is the participant.

We are using a Fetch Robot at the University of Herfordshire's Robot House (Fig. 3). The slow in, slow out velocity profile is a plugin in the robot's navigation system and is based on a trajectory roll out scheme. The Fetch robot was selected as it can move at a rate of 1 meter per second $(\mathrm{m} / \mathrm{s})$-slower than an average person's walking speed-and accelerating up to this speed takes enough time that one can notice different acceleration ramps and velocity profiles. Fetch will be holding a basket to reduce uncertainty in the handover. Fetch will be controlled partially via Wizard of $\mathrm{Oz}$, (i.e., the person in the kitchen will watch the hand over and then command Fetch to travel to the next destination), but Fetch navigates to the destination itself. For safety, both the person on the couch and the person in the kitchen will watch the robot and can activate an emergency stop switch if the robot moves off course.

\section{Preliminary RESUlts}

Pilot studies have shown that participants notice differences between the Fetch Robot moving with a linear acceleration and a robot moving with the slow in, slow out acceleration, especially when stopping. We have recorded footage of staff participating in the pilot study to be used as the video stimulus for the participant at the end of the experiment. In the ongoing main study, participants so far have commented on how they liked the smoothness of the robot's movement.

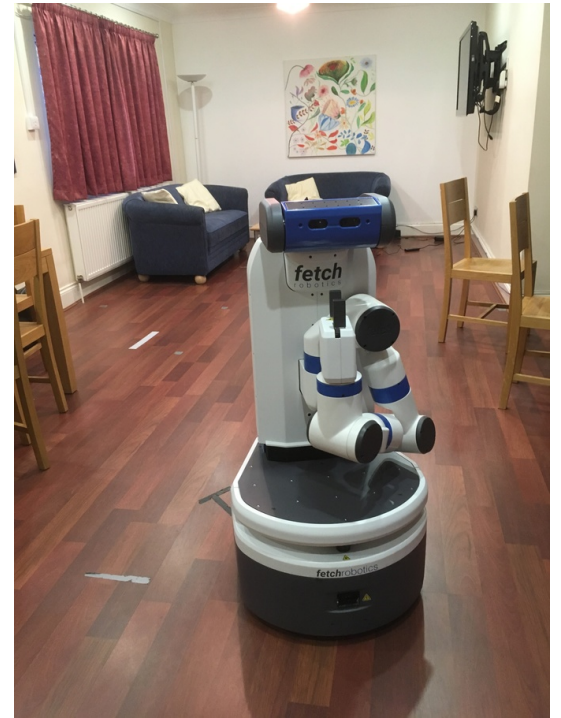

Fig. 3. The Fetch Robot used for the experiment.

\section{FUtURE DIRECTION}

We are currently recruiting participants for the study and running the experiment. We are aiming for at least 30 participants. Once we have finished the study, we will be analyzing data and writing up the results. If the results are positive, we will explore running the experiment with different kinds of robots to see if slow in, slow out velocity profile is transferable to other kinds of robots.

\section{REFERENCES}

[1] F. Thomas and O. Johnston, The Illusion of Life: Disney Animation, 1st Hyperion ed. New York: Hyperion, 1995, 575 pp., IS B N: 978-0-7868-6070-8.

[2] P. Viviani and N. Stucchi, "Biological Movements Look Uniform: Evidence of Motor-Perceptual Interactions," Journal of Experimental Psychology: Human Perception and Performance, vol. 18, no. 3, pp. 603-623, 1992. D OI: 10.1037/0096-1523.18.3.603.

[3] D. Szafir, B. Mutlu, and T. Fong, "Communication of Intent in Assistive Free Flyers," in Proceedings of the 2014 ACM/IEEE International Conference on Human-Robot Interaction, ser. HRI '14, New York, NY, USA: ACM, 2014, pp. 358-365, IS B N: 978-1-4503-2658-2. D OI: 10.1145/2559636.2559672.

[4] T. Ribeiro and A. Paiva, "The Illusion of Robotic Life: Principles and Practices of Animation for Robots," in Proceedings of the Seventh Annual ACM/IEEE International Conference on Human-Robot Interaction, ser. HRI '12, New York, NY, USA: ACM, 2012, pp. 383-390, IS B N: 978-1-4503-1063-5. DOI: $10.1145 / 2157689.2157814$.

[5] L. Takayama, D. Dooley, and W. Ju, "Expressing Thought: Improving Robot Readability with Animation Principles," in Proceedings of the 6th International Conference on HumanRobot Interaction, ser. HRI '11, New York, NY, USA: ACM, 2011, pp. 69-76, IS B N: 978-1-4503-0561-7. D OI: 10.1145/ 1957656.1957674.

[6] C. Bartneck, D. Kulić, E. Croft, and S. Zoghbi, "Measurement Instruments for the Anthropomorphism, Animacy, Likeability, Perceived Intelligence, and Perceived Safety of Robots," International Journal of Social Robotics, vol. 1, no. 1, pp. 71-81, 2009, I S S N: 1875-4791, 1875-4805. DOI: $10.1007 / \mathrm{s} 12369-008-$ 0001-3. 